\title{
Patologia Vestibular em Idade Pediátrica: Análise Retrospetiva e Revisão da Literatura
}

\section{Vestibular Disorders in the Pediatric Age: Retrospective Analysis and Review of the Literature}

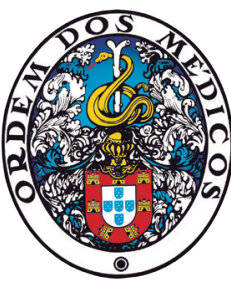

\author{
António Fontes LIMA ${ }^{1}$, Filipa Carvalho MOREIRA ${ }^{1}$, Ana Sousa MENEZES ${ }^{1}$, Isabel Esteves COSTA ${ }^{1}$, \\ Cátia AZEVEDO ${ }^{1}$, Sérgio VILARINHO ${ }^{1}$, Luís DIAS ${ }^{1}$ \\ Acta Med Port 2021 Jun;34(6):428-434 - https://doi.org/10.20344/amp.13147
}

\section{RESUMO}

Introdução: A patologia vestibular na população pediátrica é um tema bastante controverso, mas que tem ganho cada vez mais destaque. A prevalência reportada nesta população varia entre $0,7 \%$ e $15 \%$. No entanto, este valor pode estar subestimado, uma vez que a sua forma de expressão na população pediátrica é muito diferente da dos adultos, podendo ir desde a vertigem rotatória até queixas de alterações da visão, cefaleias, atraso motor ou dificuldades na aprendizagem. Embora na literatura a otite média com efusão seja considerada a principal causa de disfunção vestibular nesta faixa etária, existem outras que devem ser consideradas. O objetivo deste estudo foi descrever as características clínicas da população pediátrica encaminhada para avaliação em consulta de Otorrinolaringologia num hospital terciário, por suspeita de disfunção vestibular, desde o ano de 2013 até 2017; Também se pretendeu comparar os resultados e realizar uma revisão da literatura acerca das etiologias mais frequentes, características diagnósticas e abordagem terapêutica.

Material e Métodos: Foram analisados de forma retrospetiva os processos clínicos dos doentes observados na consulta de subespecialidade de Vertigem de Otorrinolaringologia com idades entre 0 e 18 anos. Foram excluídos os doentes com diagnóstico de otite média com efusão.

Resultados: Cumpriam os critérios de inclusão 37 doentes, dos quais $59 \%$ eram do sexo feminino, sendo a idade média da primeira consulta de 10,9 anos. O motivo mais frequente de referenciação foi a vertigem rotatória. As náuseas e cefaleias foram também sintomas frequentemente descritos. Todos os doentes foram submetidos a audiometria tonal; a videonistagmografia foi realizada em $41 \%$ dos casos; exames de imagem foram realizados em $59 \%$ dos doentes. No que respeita às etiologias do quadro, as mais frequentes foram a enxaqueca vestibular e a vertigem paroxística benigna da infância (ambas em $27 \%$ dos casos), seguida da neuronite vestibular (em 22\% dos casos).

Discussão: Embora os resultados sejam, em parte, sobreponíveis à literatura, comparativamente a outros centros de referência a diversidade de motivos de referenciação e de patologias é menor, o que pode apontar para o subdiagnóstico da disfunção vestibular nesta faixa etária.

Conclusão: A disfunção vestibular em idade pediátrica pode ter uma multiplicidade de etiologias, pelo que pediatras, neurologistas, fisiatras, médicos de família e otorrinolaringologistas devem estar alerta para as diversas formas de apresentação desta entidade. Deverão ser criados protocolos de referenciação e avaliação adequados para esta população.

Palavras-chave: Criança; Doenças Vestibulares; Neuronite Vestibular; Perturbações de Enxaqueca; Vertigem; Vertigem Paroxística Posicional Benigna

\section{ABSTRACT}

Introduction: Vestibular disorders in pediatric patients is still a controversial subject but has gained relevance over the years. In recent studies, its prevalence varied between $0.7 \%$ and $15 \%$. Nevertheless, the true prevalence can be underestimated given that its clinical presentation is expressed compared to adults; it can present as rotatory vertigo, but It can also cause vision complaints, headaches, motor delay, and learning disability. Although middle ear effusion is considered the main cause of vestibular dysfunction in this age group, other diagnoses should be considered. The aim of this study was to describe clinical features of the pediatric population referred to a subspecialist Otorhinolaryngology vertigo clinic in a tertiary hospital between 2013 and 2017 . We also aimed to compare the results and carry out a literature about the most common causes, diagnostic features and treatment approach.

Material and Methods: Clinical records of patients referred to a subspecialist Otorhinolaryngology vertigo clinic with suspicion of vestibular dysfunction aged between 0 and 18 years old were reviewed. Patients with middle ear effusion were excluded.

Results: Thirty-seven patients met the inclusion criteria. From these, $59 \%$ were female, with a mean age of 10.9 years old during the first consultation. The most common reason for referral was rotatory vertigo. Nausea and headache were also frequent complaints in our population. All patients performed audiometry; videonistagmography was performed in $41 \%$ of the cases; imaging studies were done in $59 \%$ of patients. The most common causes of vestibular dysfunction were vestibular migraine and benign paroxysmal vertigo of childhood (both in $27 \%$ of the cases), followed by vestibular neuritis (in $22 \%$ of the cases).

Discussion: Although our findings partially concur with the literature, compared with other specialist centers, the range of reasons for referral and of conditions is not as diverse, which may suggest that there is underdiagnosis of vestibular dysfunction in this age group. Conclusion: Vestibular dysfunction in the pediatric age can have several causes; pediatricians, neurologists, physiatrists, family doctors and otorhinolaryngologists must be aware of the different forms of presentation. Referral and evaluation protocols addressing pediatric patients should be created.

Keywords: Benign Paroxysmal Positional Vertigo; Child; Migraine Disorders; Vertigo; Vestibular Diseases; Vestibular Neuritis

\footnotetext{
1. Serviço de Otorrinolaringologia e Cirurgia Cérvico-facial. Hospital de Braga. Braga. Portugal.

$\triangle$ Autor correspondente: António Fontes Lima. antoniofonteslima24@gmail.com

Recebido: 17 de novembro de 2019 - Aceite: 27 de janeiro de 2020 - Online issue published: 01 de junho de 2021

Copyright $\odot$ Ordem dos Médicos 2021
} 


\section{INTRODUÇÃO}

As queixas relacionadas com disfunção vestibular (DV) são incomuns nas crianças e adolescentes, tendo uma prevalência reportada que pode variar entre $0,7 \%$ e $15 \% .{ }^{1}$ No entanto, este valor pode subestimar a verdadeira prevalência desta entidade, tendo em conta que a DV na criança tem uma expressão distinta da dos adultos. ${ }^{2}$

Os sinais e sintomas de DV em pediatria podem variar desde alterações visuais, cefaleias, descoordenação na marcha, atraso motor, dificuldade na aprendizagem, vertigem rotatória, podendo, em alguns casos, não ter qualquer manifestação clínica. Por outro lado, a relação anatómica estreita entre a cóclea e as estruturas vestibulares pode, segundo alguns autores, explicar o porquê de muitas crianças com hipoacusia sensorioneural terem algum grau de DV. ${ }^{3}$

Aliado à dificuldade em interpretar os sintomas, o próprio exame físico nesta população tem limitações (nomeadamente no que respeita à colaboração), o que pode, em alguns casos, levar a diagnósticos erróneos, ou à ausência de diagnóstico. ${ }^{1-3}$

Os exames complementares de diagnóstico (ECD) utilizados em adultos, embora possam ser, na sua maioria, utilizados para avaliação em idade pediátrica, necessitam de adaptações não apenas em termos de execução dos mesmos, mas também relativamente a valores de normalidade. ${ }^{2,4}$

No entanto, e apesar da evolução tecnológica no desenvolvimento de meios complementares para diagnóstico, a história clínica e o exame objetivo são o ponto de partida fundamental para estabelecer uma hipótese. As hipóteses de diagnóstico em idade pediátrica são diferentes das do adulto, havendo algumas patologias que são exclusivas nesta idade..$^{1,3,5}$

Embora a otite média com efusão seja descrita na literatura como a principal causa de DV nesta faixa etária, existem outras causas que devem ser consideradas. Essas outras causas de vertigem na infância são, segundo a literatura, a vertigem paroxística benigna da infância (VPBI), a enxaqueca vestibular (EV) e o traumatismo crânio-encefálico (TCE) ${ }^{1,5}$ Causas menos comuns são a neuronite vestibular (NV), labirintite, vertigem paroxística posicional benigna (VPPB), a doença de Ménière, e tumores do sistema nervoso central. A vertigem psicogénica também deve ser tida em conta. ${ }^{5}$

O objetivo deste estudo é a análise dos casos de doentes em idade pediátrica referenciados à consulta de subespecialidade de Vertigem num hospital terciário, esclarecendo formas de apresentação, achados no exame físico e nos ECD realizados, e as etiologias que mais frequentemente foram observadas nesta consulta. Posteriormente, pretende-se fazer uma reflexão acerca dos mesmos, revendo a literatura mais pertinente e também mais atual sobre o tema.

\section{MATERIAL E MÉTODOS}

Foi realizado um estudo retrospetivo para análise de uma série de casos. Foram avaliados os doentes em idade pediátrica (considerada, para o efeito, a idade compreendida entre os 0 e 17 anos e 364 dias) que foram referenciados à consulta de subespecialidade de Vertigem no serviço de Otorrinolaringologia no Hospital de Braga (HB), entre os anos de 2013 e 2017. Foram recolhidos os seguintes dados: género, idade do início da sintomatologia, história clínica detalhada, sintomas major à apresentação, exame otoneurológico (presença de nistagmo espontâneo sob lunetas de Frenzel, teste de impulso cefálico (TIC), oculomotricidade, Romberg, manobra de Unterberger-Fukuda (UF) e presença de nistagmo posicional), resultado dos exames de avaliação cócleo-vestibular realizados (audiometria tonal e videonistagmografia), exames de imagem [tomografia computorizada (TC) e/ou ressonância magnética (RM)] e o diagnóstico.

Foram excluídos deste estudo, doentes com o diagnóstico de otite média com efusão (OME).

O protocolo de estudo foi aprovado pela Comissão de Ética para a Saúde do HB.

\section{RESULTADOS}

Cumpriram os critérios de inclusão 37 doentes. Os resultados são apresentados nas Tabelas 1 e 2 . Entre os doentes incluídos, 59\% $(n=22)$ eram do sexo feminino. A idade média da primeira consulta foi 10,9 anos $\pm 3,7$, tendo variado entre os quatro e 17 anos.

O sintoma que motivou a referenciação em todos os doentes foi a vertigem rotatória. Os sintomas associados mais frequentemente descritos foram náuseas, em $46 \%$ ( $n$ $=17)$, seguido de cefaleia $(35 \%, n=13)$, vómitos $(24 \%, n$ = 9), distúrbios visuais $(16 \% \mathrm{n}=6)$ e perda transitória de audição $(5 \%, n=2)$.

O exame físico revelou alterações diversas que foram essenciais para elaboração do diagnóstico mais provável: nistagmo espontâneo foi observado em $16 \%$ dos doentes; nistagmo posicional também foi observado em $16 \%$ dos casos; o TIC foi positivo em $27 \%$ dos doentes, o UF revelou um desvio significativo em $24 \%$ e o teste de Romberg mostrou instabilidade e/ou queda em $21 \%$ dos doentes.

Em termos de ECD, todos os doentes realizaram audiometria tonal, mostrando-se normal em todos eles, com média do limiar tonal médio da via aérea à direita de 12,1 décibeis $(\mathrm{dB}) \pm 4,3$ e $12,7 \mathrm{~dB} \pm 5,7$ à esquerda.

A videonistagmografia foi realizada em $41 \%$ dos doentes $(n=15)$, mostrando alterações em quatro casos, nomeadamente achados compatíveis com hipofunção vestibular unilateral.

Exames de imagem como TC e/ou RM foram realizados em $59 \%$ dos doentes; apenas foram encontradas alterações num caso.

Os diagnósticos mais frequentes nesta população foram a EV e a VPBI, ambas em $27 \%$ dos casos, seguida pela $\mathrm{NV}$, em $22 \%$, e pela VPPB, em $16 \%$.

A EV foi observada predominantemente em doentes do sexo feminino, com idade média da primeira consulta de 12 anos $\pm 1,6$. Nestes doentes, além da vertigem, todos 
Tabela 1 - Características da população pediátrica à apresentação

\begin{tabular}{|c|c|c|}
\hline Idade média à apresentação & 10,89 anos & \\
\hline Género (Feminino/ Masculino) & $59 \%(n=22) / 41 \%(n=15)$ & \\
\hline \multirow[t]{6}{*}{ Sintomas à apresentação } & Vertigem rotatória & $100 \%(n=37)$ \\
\hline & Náuseas & $46 \%(n=17)$ \\
\hline & Cefaleias & $35 \%(n=13)$ \\
\hline & Vómitos & $24 \%(n=9)$ \\
\hline & Alterações visuais & $16 \%(n=6)$ \\
\hline & Hipoacusia & $5 \%(n=2)$ \\
\hline \multirow[t]{5}{*}{ Achados patológicos no exame-otoneurológico } & Nistagmo espontâneo & $16 \%(n=6)$ \\
\hline & TIC positivo & $27 \%(n=10)$ \\
\hline & Romberg positivo & $21 \%(n=8)$ \\
\hline & Unterberger-Fukuda com desvio ou rotação & $24 \%(n=9)$ \\
\hline & Nistagmo posicional & $16 \%(n=6)$ \\
\hline \multirow{3}{*}{$\begin{array}{l}\text { ECDs: doentes que realizaram o exame/ doentes } \\
\text { que tiveram alterações no exame }\end{array}$} & Audiograma tonal e vocal & $100 \%(n=37) / 0 \%$ \\
\hline & Videonistagmografia & $41 \%(n=15) / 10 \%(n=4)$ \\
\hline & TC ou RM & $59 \%(n=22) / 2,7 \%(n=1)$ \\
\hline
\end{tabular}

Tabela 2 - Diagnósticos, idade média da primeira consulta

\begin{tabular}{llcc}
\hline & $\%$ & $\mathbf{n}$ & Idade \\
\hline Enxaqueca vestibular & $27 \%$ & 10 & 12,0 anos \\
Vertigem paroxística benigna da infância & $27 \%$ & 10 & 5,8 anos \\
Neuronite vestibular & $22 \%$ & 8 & 14,4 anos \\
Vertigem paroxística posicional benigna & $16 \%$ & 6 & 13,0 anos \\
Psicogénica & $5 \%$ & 2 & 14,5 anos \\
Esclerose múltipla & $3 \%$ & 1 & 16,0 anos \\
\hline
\end{tabular}

se queixavam de cefaleia; $70 \%$ queixavam-se de náuseas; $40 \%$ doentes queixavam-se de alterações visuais antes ou durante a crise. O exame objetivo na consulta era normal em todos os casos. Em termos de ECD, apenas num paciente se verificaram alterações na VNG, compatíveis com uma hipofunção vestibular unilateral; todos realizaram exame de imagem (solicitados por ORL ou por Neuropediatria). O controlo sintomático no momento das crises era feito com analgésicos e anti-eméticos. Apenas em dois casos, nos quais as crises eram frequentes (mais do que uma vez por semana), eram utilizados fármacos profiláticos, nomeadamente flunarizina (na dose de $5 \mathrm{mg} / \mathrm{dia}$ ) e amitriptilina (na dose de $1 \mathrm{mg} / \mathrm{kg} / \mathrm{dia}$ ), com bons resultados. Todos os doentes deste grupo eram simultaneamente seguidos na consulta de Neuropediatria.

A VPBI foi um diagnóstico comum. A idade média da primeira consulta foi 5,8 anos $\pm 1,7$ (mínimo de quatro anos, e máximo de oito anos). Nenhum dos doentes foi observado durante a crise; o exame físico e cócleo-vestibular foi normal em todos os casos. Apenas um realizou exame de imagem (prévio ao encaminhamento para a consulta de ORL), que se mostrou normal. Estes doentes foram seguidos entre três e quatro anos, tendo-se verificado uma diminuição da frequência de crises com o tempo, e posterior resolução do quadro, com ausência de crises durante mais de um ano em todos os casos antes da alta.
A NV foi também um motivo frequente de observação nesta consulta. A idade média ao diagnóstico foi de 14 anos $\pm 2,2$. Na nossa população, numa primeira observação, e após diagnóstico clínico, todos os doentes foram internados sob corticoterapia sistémica pela sintomatologia intensa; aquando de melhoria clínica, tiveram alta e foram encaminhados para consulta de Vertigem. Todos foram, nessa fase, submetidos a exame de imagem; este não mostrou alterações relevantes em nenhum deles. Posteriormente, já após resolução da fase aguda, foram submetidos a provas calóricas, que mostraram recuperação da hipofunção vestibular em todos os casos, exceto num, o qual foi orientado para reabilitação em plataforma de posturografia, por manter alterações do equilíbrio.

Relativamente à VPPB, os achados ao exame físico foram fundamentais: um dos casos tratou-se de uma VPPB do canal semicircular (CSC) lateral, identificada com a manobra de McClure; outro dos casos correspondeu a uma VPPB do CSC anterior, com nistagmo na manobra de Rose (head hanging); os restantes casos trataram-se de VPPB do CSC posterior, com nistagmo identificado na manobra de Dix-Hallpike. Na nossa população, utilizou-se a manobra de Lempert para a VPPB do CSC lateral; a manobra de Yacovino para a VPPB do CSC anterior; e a manobra de Epley para os casos de VPPB do CSC posterior.

Incluíram-se ainda dois casos de adolescentes do sexo 
feminino e com antecedentes de patologia psiquiátrica que foram enviadas para a consulta por quadro que identificavam como vertigem, e que ocorria sobretudo em situações de ansiedade; após avaliação, na qual o exame físico se mostrou normal, o exame cócleo-vestibular sem alterações, e RM normal, o seu quadro foi classificado como vertigem psicogénica.

Finalmente, um dos casos revistos referia-se a um adolescente de 16 anos. que foi encaminhado para a consulta de Vertigem após ida à urgência. Nessa altura, tinha um quadro compatível com uma síndrome vestibular agudo, com vertigem, náuseas e vómitos associados; no entanto, este adolescente tinha também história prévia de episódio de hemiparesia dos membros superior e inferior direitos, de etiologia indeterminada e de resolução espontânea. No exame físico tinha nistagmo espontâneo horizonto-rotatório grau 2, TIC sem sacada de refixação e restante exame otoneurológico normal; foi solicitado RM, na qual se verificaram alterações sugestivas de doença desmielinizante, que posteriormente foi confirmado tratar-se de esclerose múltipla.

\section{DISCUSSÃO}

Apesar da literatura mostrar que a DV em idade pediátrica pode ter diversas formas de apresentação, na nossa população o principal sintoma que motivou o encaminhamento para consulta foi a vertigem rotatória, queixa de todos os doentes. Szirmai também relatou a vertigem como o sintoma que mais frequentemente motivou a observação de doentes pediátricos com suspeita de DV. ${ }^{6}$ Por outro lado, sintomas frequentemente associados foram as náuseas, vómitos, e as cefaleias, o que também foi verificado em estudos semelhantes. ${ }^{6-8}$

O facto do motivo de referenciação na nossa população ser pouco diverso pode dever-se ao desconhecimento das formas de apresentação da patologia vestibular nesta idade por parte dos médicos que encaminham os doentes para a consulta, associando-a apenas à vertigem rotatória. No entanto, se a vertigem é típica de hipofunção vestibular unilateral periférica aguda, nos casos de hipofunção crónica, este sintoma não é frequente. ${ }^{9} \mathrm{~A}$ literatura mais recente mostra que, além da vertigem, a DV em idade pediátrica apresenta-se muitas vezes como desequilíbrio, atraso no desenvolvimento motor, ou comprometimento visual. Em idades ainda mais precoces, comportamentos como agarrar-se aos pais persistentemente, pedir para ser segurado, recusa a levantar ou quedas frequentes podem ser sinais de DV. $2,5,9,10$

Ao contrário do que acontece na nossa população, no que respeita a centros de referência, verifica-se uma realidade bastante diversa relativamente aos motivos que levam os médicos assistentes a suspeitar de DV e, por outro lado, a encaminhar para avaliação do sistema vestibular por ORL: O'Reilly et al referem o atraso de desenvolvimento motor como um dos principais motivos de referenciação à consulta de Vertigem Pediátrica por parte de pediatras do desenvolvimento, fisiatras e neurologistas; por outro lado, crianças com síndromes que podem estar associados a alterações vestibulares (tais como a síndrome de Usher, Waardenburg, etc) são avaliadas de forma sistemática nesta consulta; finalmente, todas as crianças com hipoacusia sensorioneural (HSN) comprovada são submetidas a estudo completo do sistema vestibular, dado que, segundo estudos recentes, dependendo da etiologia e grau de surdez, entre $38 \%$ - $91 \%$ das crianças com HSN têm algum tipo de DV; exemplos do último são os inúmeros protocolos de avaliação pré-implante coclear que incluem, hoje em dia, a avaliação do sistema vestibular. 2,3,11

A avaliação vestibular em idade pediátrica pode ser complexa por uma série de razões: nesta idade, a capacidade de auto-perceção de sintomas ou a identificação destes como anormais pode ser difícil; por outro lado, a falta de colaboração no exame físico e nos ECD compromete, por vezes, a sua realização. ${ }^{2-5}$ No entanto, o exame físico, incluindo uma adequada avaliação otoneurológica, é essencial para estabelecer as hipóteses diagnósticas mais prováveis e, assim, determinar qual a melhor atitude diagnóstica e terapêutica.

Uma avaliação cócleo-vestibular completa é importante quando existe este tipo de sintomas. A audiometria tonal, acompanhada da impedancimetria, são relevantes para o diagnóstico diferencial deste tipo de queixas. Por outro lado, as provas calóricas (integradas na videonistagmografia) são, ainda hoje, consideradas o gold-standard de avaliação do sistema vestibular. ${ }^{9,12}$ Este exame providencia informação acerca do ramo superior do nervo vestibular e do canal semicircular horizontal, e é sensível mesmo para disfunções vestibulares ligeiras. ${ }^{12}$ No entanto, a compliance sobretudo em crianças com menos de seis anos, é bastante questionável, uma vez que implica atenção do examinado e pode ser desconfortável para a criança, uma vez que implica irrigação do canal auditivo externo. 2,9,12

No nosso departamento, o estudo desta população tem-se baseado nos exames acima referidos, uma vez que eram os únicos disponíveis no intervalo temporal estudado. Todavia, ainda no âmbito da avaliação objetiva da população pediátrica, alguns exames têm ganho importância, sendo utilizados em diversos centros especializados. Dhont et al descrevem protocolos de avaliação objetiva de sintomas vestibulares que variam segundo a idade das crianças e que incluem, além dos descritos, o video-head impulse test (vHIT), potenciais evocados miogénicos vestibulares cervicais (cVEMP) e oculares (oVEMP). ${ }^{2}$ Estes exames permitem uma avaliação exaustiva e específica do sistema vestibular - o vHIT permite a avaliação de cada um dos canais semicirculares; os cVEMP permitem a avaliação do sáculo e os oVEMP do utrículo., ${ }^{9,12}$ No conjunto, os exames permitem a avaliação de todos os órgãos-terminais do sistema vestibular. Embora a metodologia de realização dos exames implique adaptações para a idade pediátrica, diversos autores usam-nos a partir dos seis meses de vida. . $^{2,12}$

Relativamente aos exames de neuroimagem, estes são de valor questionável..$^{13} \mathrm{~A}$ literatura mostra-nos que a TC é raramente útil (poderá ter utilidade, por exemplo, quando 
há suspeita de fratura do osso temporal pela sua melhor definição óssea); por outro lado, a RM é o exame de eleição quando se pretendem avaliar estruturas tais como a fossa posterior ou o ouvido interno.,13 Além disso, exames de imagem podem ter indicação em doentes com vertigem e défices neurológicos associados ou cefaleia persistente..$^{13}$ No entanto, estes exames, além de dispendiosos, exigem a colaboração da criança, ou apoio de Anestesiologia para a sua realização (para sedação ou anestesia geral). No caso da nossa população, embora uma grande parte dos doentes os tenha realizado, apenas num se encontraram alterações compatíveis com doença desmielinizante, que mais tarde se confirmou tratar de esclerose múltipla. A elevada percentagem de doentes que realizou estes exames na nossa população explica-se pelos grupos de diagnosticados com EV e com NV, que representam, em conjunto, aproximadamente $50 \%$ dos doentes, todos eles submetidos a exame de imagem.

$\mathrm{Na}$ nossa população, os diagnósticos mais frequentemente observados foram a EV, VPBI, NV e a VPPB. Estes resultados são comparáveis a vários autores, com a $E V$ e a VPBI como as causas mais frequentes de DV em idade pediátrica na maioria dos estudos..$^{6-8,14,15}$ As diferenças percentuais podem dever-se a diferentes metodologias, critérios de inclusão e exclusão; por exemplo, alguns estudos incluíram apenas a população pediátrica com queixa principal de vertigem ${ }^{6,8,15}$; por outro lado, O’Reilly et al incluíram toda a população com menos de 18 anos encaminhada para a consulta de distúrbios vestibulares em crianças, independentemente do sintoma principal. ${ }^{16}$ Além disso, a maioria dos estudos excluiu também os casos de OME, dada a grande variabilidade na apresentação de doentes com DV nesses casos. $6,8,14,15$

\section{Enxaqueca vestibular}

O diagnóstico de EV está assente em critérios bem definidos pela International Headache Society (Tabela 3$) .{ }^{17} \mathrm{Se}-$ gundo Davitt et al, esta patologia poderá ser responsável por até $35 \%$ da população pediátrica com queixas de vertigem, assumindo menor importância em adultos (responsável por menos de $7 \%$ dos adultos com estas queixas)..$^{18}$ Estes dados são corroborados na nossa série de casos, que identificou a EV como uma das causas mais frequentes.

Por outro lado, a sua fisiopatologia é ainda bastante controversa. ${ }^{1}$ Habitualmente manifesta-se com sintomas vestibulares recorrentes acompanhadas com enxaqueca (antes, durante ou depois), com duração entre cinco minutos e 72 horas; algumas crianças também têm queixas de foto e fonosenssibilidade. Entre as crises, além de assintomáticos, habitualmente o exame físico é normal. ${ }^{5}$

Embora a primeira linha de tratamento nesta idade seja não farmacológica (através da evicção de triggers), existem opções farmacológicas. Durante a crise, o tratamento sintomático é muitas vezes suficiente, com analgésicos e anti-eméticos. O restante tratamento farmacológico pode ser subdividido em abortivo, utilizado durante a crise aguda, e profilático; deve ser oferecido quando as crises são frequentes, uma vez por semana ou mais, ou quando são debilitantes ou prolongadas, e de acordo com a preferência do cuidador. ${ }^{1,5,10,18}$ Os bloqueadores de canais de cálcio e os triptanos são os mais frequentemente utilizados; anticonvulsivantes, como o topiramato e a gabapentina, e tricíclicos, como a amitriptilina, também parecem ser eficazes na profilaxia das crises; no entanto, uma vez que não existem estudos acerca do uso desses fármacos em idade pediátrica, o tratamento tem sido extrapolado a partir de guidelines de adultos. ${ }^{5}$

\section{Vertigem paroxística benigna da infância}

A VPBI mostrou-se um diagnóstico frequente na nossa consulta $(27 \%)$. Noutros estudos, foi responsável por entre $12 \%-26 \%$ da população pediátrica com queixas de vertigem. ${ }^{1,5}$ Trata-se de uma patologia cuja sintomatologia habitualmente se inicia em idades precoces, entre um e quatro anos de idade - o que foi verificado na nossa população; tem, usualmente, um curso auto-limitado, e raramente se observa depois dos oito anos. ${ }^{1}$ Por isso mesmo, o tratamento é sintomático (com anti-eméticos), se necessário; deve ainda ser explicado aos pais a natureza benigna desta entidade. ${ }^{5}$

Caracteriza-se por episódios súbitos de vertigem, de início inesperado, sem pródromos que podem durar segundos a minutos; pode ser acompanhada, nesta fase, por

Tabela 3 - Critérios diagnósticos de enxaqueca vestibular ${ }^{17}$

\section{Enxaqueca vestibular}

A. Pelo menos 5 episódios de sintomas vestibulares de intensidade moderada ou severa, com duração de 5 minutos a 72 horas

B. História atual ou prévia de enxaqueca com ou sem aura

C. Um ou mais sintomas em pelo menos $50 \%$ dos episódios:

- enxaqueca com pelo menos duas características: unilateral, pulsátil, dor de intensidade moderada ou severa, agravamento por atividade física rotineira

- fotofobia ou fonofobia

- aura visual

D. Não é melhor explicado por outro diagnóstico vestibular ou de enxaqueca

\section{Enxaqueca vestibular provável}

A. Pelo menos 5 episódios de sintomas vestibulares de intensidade moderada ou severa, com duração de 5 minutos a 72 horas

B. Apenas um dos critérios B e C para enxaqueca vestibular é cumprido (história de enxaqueca ou sintomas de enxaqueca durante o episódio)

C. Não é melhor explicado por outro diagnóstico vestibular ou de enxaqueca 
nistagmo espontâneo. ${ }^{5}$ É considerada uma patologia de origem central, do espectro da enxaqueca. ${ }^{17}$ Existindo alguma sobreposição entre a VPBI e a EV, alguns autores admitem que a primeira poderá, na verdade, ser um precursor da segunda. $5,7,15,17$

\section{Vertigem paroxística posicional benigna}

A VPPB é uma entidade rara em idade pediátrica. Este facto é atribuído, por alguns autores à hipótese de, nesta idade, as otocónias se encontrarem firmemente aderidas à mácula do utrículo, o que desfavorece o aparecimento desta entidade. ${ }^{19}$ Por outro lado, em idade adulta é uma das causas mais frequentes de vertigem. Ainda assim, na nossa população, esta patologia mostrou-se bastante frequente. O tratamento da VPPB passa por manobras de reposicionamento.

\section{Neuronite vestibular}

Embora a NV seja uma entidade relativamente rara, está entre as mais frequentes causas de vertigem rota-

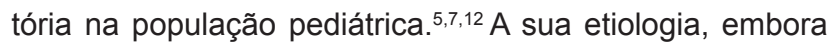
controversa, parece associar-se a uma infeção viral prévia (na maioria das vezes, uma infeção das vias aéreas superiores). ${ }^{1}$ A apresentação é semelhante à dos adultos, quer em termos de sintomatologia, com vertigem rotatória persistente, dificuldade na deambulação, náuseas e vómitos, quer em termos de exame físico, com sinais de hipofunção vestibular aguda (com nistagmo espontâneo, TIC com sacada de refixação presente, e com alterações no teste de Romberg e UF).

O tratamento é discutível, não estando estabelecida uma abordagem gold-standard. ${ }^{5,9}$ Baseia-se, habitualmente, em corticoterapia sistémica e reabilitação vestibular, à semelhança da população adulta; por outro lado, o papel dos anti-virais ainda não está estabelecido.,

Comparativamente aos adultos, o potencial de recuperação em idade pediátrica é bastante mais favorável. ${ }^{1,5,7}$

\section{Outras causas}

O TCE é descrito na literatura como uma causa relativamente frequente de queixas de instabilidade ou vertigem. ${ }^{9}$ Os mecanismos fisiopatológicos são diversos, mas podem estar relacionados com fratura do osso temporal, concussão do labirinto ou, mais raramente, fístula perilinfática. Um dos casos descritos de VPPB na nossa população surgiu após TCE grave.

A doença de Ménière caracteriza-se por episódios de vertigem rotatória, acompanhados de hipoacusia flutuante, acufeno e sensação de plenitude aural. ${ }^{5}$ Segundo o consenso entre várias sociedades dedicadas ao estudo do equilíbrio elaborado em 2015, o diagnóstico definitivo implica pelo menos dois episódios com os sintomas descritos com duração entre 20 minutos e 12 horas (Tabela 4)..$^{20}$ Embora a doença seja mais frequente em idade adulta, entre os 30 e 50 anos, em algumas séries foi responsável entre $1,5 \%$ e $4 \%$ da vertigem em idade pediátrica. ${ }^{1}$

Finalmente, a OME é tida, na literatura, como a causa mais frequente de sintomas vestibulares em idade pediátrica. ${ }^{1,2,5,10,18} \mathrm{Na}$ maior parte das vezes, esta entidade não se associa a vertigem propriamente dita, sendo mais frequentemente reportada pelos pais como instabilidade na marcha, marcha 'desajeitada', ou quedas frequentes. ${ }^{1}$ No entanto, na maior parte das vezes, as queixas resolvem após miringotomia com colocação de tubo de ventilação transtimpânico (TVT). Na série de 136 crianças com OME e queixas vestibulares avaliada por Golz et al, 96\% resolveram os sinais de alterações vestibulares após colocação de TVT. ${ }^{21}$

\section{CONCLUSÃO}

Os resultados obtidos relativamente à consulta de subespecialidade de Vertigem do HB foram semelhantes aos estudos com metodologia idêntica disponível. A história clínica e o exame físico podem ser suficientes para estabelecer o diagnóstico, sendo os exames cócleo-vestibulares e a neuroimagem de importância variável devendo, portanto, ser adequados caso-a-caso.

Por outro lado, a patologia vestibular na população pediátrica tem assumido cada vez maior importância uma vez que se admite, de acordo com a literatura mais recente, que seja mais prevalente que o considerado no passado, sobretudo pelas diversas formas de apresentação. O reconhecimento dos diferentes sinais e sintomas por parte dos profissionais que observam doentes em idade pediátrica é fundamental para uma adequada referenciação, para diagnóstico e para o tratamento. Os nossos resultados, embora sejam semelhantes à literatura, mostram pouca variabilidade em termos de motivos de referenciação, o que leva os autores a inferir algum desconhecimento por parte da comunidade médica acerca da DV em idade pediátrica.

Tabela 4 - Critérios diagnósticos de doença de Menière ${ }^{20}$

1. Doença de Menière definitivo

A. Dois ou mais episódios de vertigem rotatória espontânea, cada um com duração entre 20 minutos a 12 horas

B. Documentação audiométrica de surdez sensorioneural nas baixas a médias frequências num ouvido em pelo menos uma ocasião, durante ou depois de um episódio de vertigem

C. Sintomas auditivos flutuantes (hipoacusia, tinnitus ou plenitude) no ouvido afetado

D. Não é melhor explicado por outro diagnóstico vestibular

2. Doença de Menière provável

A. Dois ou mais episódios de vertigem rotatória espontânea, cada um com duração entre 20 minutos a 12 horas

B. Sintomas auditivos flutuantes (hipoacusia, tinnitus ou plenitude) no ouvido afetado

C. Não é melhor explicado por outro diagnóstico vestibular ou de enxaqueca 
Consequentemente, isto pode conduzir a uma taxa de diagnóstico e terapêutica abaixo do desejável.

De acordo com a literatura atual, é recomendada a criação de guidelines de referenciação para avaliação vestibular de doentes que tenham diagnóstico de síndromes comummente associados a DV e, por outro lado, de crianças com diagnóstico de surdez sensorioneural. Finalmente, é necessária a implementação de protocolos de avaliação destes doentes, nomeadamente através da inclusão de exames objetivos com maior acuidade diagnóstica, que, com as devidas adaptações, ultrapassem as dificuldades de execução e interpretação nesta idade, e, por outro lado, a criação de equipas multidisciplinares preparadas para o tratamento destes doentes.

\section{OBSERVAÇÕES}

Este trabalho foi apresentado na mesa redonda intitulada "Vestibular testing in children" no $5^{\circ}$ Congresso da Confederation of European Otorhinolarynology and Head and Neck Surgery, realizado em julho de 2019, em Bruxelas, Bélgica.

\section{REFERÊNCIAS}

1. Gioacchini FM, Alicandri-Ciufelli M, Kaleci S, Magliulo G, Re M. Prevalence and diagnosis of vestibular disorders in children: a review. Int J Pediatr Otorhinolaryngol. 2014;78:718-24.

2. Dhondt C, Dhooge I, Maes L. Vestibular assessment in the pediatric population. Laryngoscope. 2019;129:490-3.

3. O'Reilly RC, Greywoode J, Morlet T, Miller F, Henley J, Church C, et al. Comprehensive vestibular and balance testing in the dizzy pediatric population. Otolaryngol Head Neck Surg. 2011;144:142-8.

4. Kuhn JJ, Lavender VH, Hunter LL, McGuire SE, Meinzen-Derr J, Keith RW, et al. Ocular vestibular evoked myogenic potentials: normative findings in children. J Am Acad Audiol. 2018;29:443-50.

5. Devaraja K. Vertigo in children; a narrative review of the various causes and their management. Int J Pediatr Otorhinolaryngol. 2018;111:32-8.

6. Szirmai A. Vestibular disorders in childhood and adolescents. Eur Arch Oto-Rhino-Laryngol. 2010;267:1801-4.

7. Gruber M, Cohen-Kerem R, Kaminer M, Shupak A. Vertigo in children and adolescents: characteristics and outcome. Scientific World Journal. 2012;2012:109624

8. Erbek SH, Erbek SS, Yilmaz I, Topal O, Ozgirgin N, Ozluoglu LN, et al. Vertigo in childhood: a clinical experience. Int J Pediatr Otorhinolaryngol. 2006;70:1547-54

9. Rine RM, Wiener-Vacher S. Evaluation and treatment of vestibular dysfunction in children. Neuro Rehabilitation. 2013;32:507-18.

10. Casani AP, Dallan I, Navari E, Sellari Franceschini S, Cerchiai N. Vertigo in childhood: proposal for a diagnostic algorithm based upon clinical experience. Acta Otorhinolaryngol Ital. 2015;35:180-5.

11. Martens S, Dhooge I, Dhondt C, Leyssens L, Sucaet M, Vanaudenaerde

\section{PROTEÇÃO DE PESSOAS E ANIMAIS}

Os autores declaram que os procedimentos seguidos estiveram de acordo com os regulamentos estabelecidos pelos responsáveis da Comissão de Investigação Clínica e Ética e de acordo com a Declaração de Helsínquia da Associação Médica Mundial, actualizada em 2013.

\section{CONFIDENCIALIDADE DOS DADOS}

Os autores declaram ter seguido os protocolos do seu centro de trabalho acerca da publicação de dados.

\section{CONFLITOS DE INTERESSE}

Os autores declaram não ter qualquer conflito de interesse relativamente ao presente artigo.

\section{FONTES DE FINANCIAMENTO}

Os autores declaram que este estudo foi realizado sem recurso a subsídio ou bolsa.

S, et al. Vestibular Infant Screening - Flanders: the implementation of a standard vestibular screening protocol for hearing-impaired children in Flanders. Int J Pediatr Otorhinolaryngol. 2019;120:196-201.

12. Janky KL, Rodriguez Al. Quantitative vestibular function testing in the pediatric population. Semin Hear. 2018;39:257-74.

13. Niemensivu R, Pyykkö I, Valanne L, Kentala E. Value of imaging studies in vertiginous children. Int J Pediatr Otorhinolaryngol. 2006;70:1639-44.

14. Wiener-Vacher SR. Vestibular disorders in children. Int $\mathrm{J}$ Audiol. 2008;47:578-83.

15. Niemensivu R, Pyykkö I, Kentala E. Vertigo and imbalance in children Arch Otolaryngol Neck Surg. 2005;131:996.

16. O'Reilly RC, Morlet T, Nicholas BD, Josephson G, Horlbeck D, Lundy $\mathrm{L}$, et al. Prevalence of vestibular and balance disorders in children. Otol Neurotol. 2010;31:1441-4.

17. Lempert T, Olesen J, Furman J, Waterston J, Seemungal B, Carey J, et al. Vestibular migraine: diagnostic criteria. J Vestib Res. 2012;22:16772.

18. Davitt M, Delvecchio MT, Aronoff SC. The differential diagnosis of vertigo in children. Pediatr Emerg Care. 2017;00:1.

19. Baloh RW, Honrubia V. Childhood onset of benign positional vertigo Neurology. 1998;50:1494-6.

20. Lopez-Escamez JA, Carey J, Chung WH, Goebel JA, Magnusson M, Mandalà M, et al. Diagnostic criteria for Menière's disease. J Vestib Res Equilib Orientat. 2015;25:1-7.

21. Golz A, Netzer A, Angel-Yeger B, Westerman ST, Gilbert LM, Joachims $\mathrm{HZ}$. Effects of middle ear effusion on the vestibular system in children. Otolaryngol Head Neck Surg. 1998;119:695-9. 